This item was submitted to Loughborough's Research Repository by the author.

Items in Figshare are protected by copyright, with all rights reserved, unless otherwise indicated.

\title{
Spatiotemporal processing in neural networks with random synaptic
} background activity

PLEASE CITE THE PUBLISHED VERSION

PUBLISHER

(C) American Physical Society

LICENCE

CC BY-NC-ND 4.0

REPOSITORY RECORD

Bressloff, P.C.. 2019. "Spatiotemporal Processing in Neural Networks with Random Synaptic Background Activity”. figshare. https://hdl.handle.net/2134/1715. 


\title{
Spatiotemporal processing in neural networks with random synaptic background activity
}

\author{
Paul C. Bressloff \\ Department of Mathematical Sciences, Loughborough University of Technology, Loughborough, \\ Leicstershire LE11 3TU, United Kingdom
}

(Received 10 January 1995)

\begin{abstract}
We establish a formal similarity between a neural network with random synaptic background activity and a physical system in a heat bath. We then use techniques from nonequilibrium statistical physics to evaluate the average linear response of a lateral inhibitory network in a random background. We show how averaging over the background leads to nontrivial frequency-dependent modifications in network response.

PACS number(s): 87.10. $+\mathrm{e}, 02.50 .-\mathrm{r}, 05.20 .-\mathrm{y}$
\end{abstract}

\section{INTRODUCTION}

A number of recent experimental studies have established that background synaptic activity can influence the behavior of cortical neurons [1-3]. The underlying mechanism is due to shunting, that is, increases in the conductance of synapses induced by such activity leads to local changes in the membrane time constant [4]; this holds for both inhibitory and excitatory synapses. Indeed, variations in the level of background activity can reduce the effective time constant $\tau$ by a factor of 10 . This has a number of important consequences:

(i) For small values of $\tau$, a neuron tends to act like a coincidence detector whereas for larger values it performs the role of a temporal integrator. Thus background activity could selectively determine the particular mode of operation carried out by a neuron.

(ii) Spatially nonuniform patterns of background activity impinging on the dendritic tree of a neuron results in a corresponding nonuniform modulation of the local membrane time constant of the tree. This could, for example, influence the effectiveness of distal dendrites at certain locations to affect events at the cell body or soma [1].

(iii) In the presence of constant inputs, reduction in the membrane time constant due to background activity leads to a corresponding reduction in the steady-state membrane potential of a neuron, which in turn produces a lower firing rate [5].

(iv) Random synaptic background activity is a source of multiplicative noise.

In this paper, we use techniques from nonequilibrium statistical physics to analyze the effects of multiplicative noise arising from synaptic background activity (point iv) on spatiotemporal processing in lateral inhibitory networks. We begin by considering a single leaky-integrator neuron with background activity modeled as a multicomponent dichotomous colored noise process (Sec. II). Solving for the membrane potential using a variation of parameters formula and averaging over the background leads to a quantity analogous to the relaxation function of a randomly modulated oscillator [6]. Following Refs. $[7,8]$, we calculate the average response of the neuron using the time convolution generalized master equation for- mulation [9] and the method of partial cumulants [10,11]. We find that in the case of a constant external input, a fluctuating background leads to a smaller reduction in the membrane time constant than a constant background of the same average intensity. We also analyze the neuron's average response to sinusoidal inputs. The fluctuating background now results in a frequency-dependent modification in the effective membrane time constant leading to nontrivial changes in both the phase and amplitude of response. We show how this affects the temporal low-pass filtering properties of a leaky-integrator neuron.

Having discussed the single-neuron case, we then turn to spatiotemporal processing in lateral inhibitory networks (LIN's). These networks have been studied in a range of biological systems, particularly those concerned with sensory perception. Examples are the Limulus compound eye [12], the mammalian retina $[13,14]$, the somatosensory system [15], and the auditory system [16]. In most of these systems, lateral inhibition serves to sharpen spatial input patterns, that is, highlighting edges and peaks. When nonlinearities associated with the firing mechanism of a neuron are included lateral inhibitory networks can exhibit more complex behavior such as hysteresis phenomena and pattern formation $[17,18]$. In this paper, however, we concentrate on linear models since this allows us to carry over the analysis of the singleneuron case. It should also be noted that the linear approximation is reasonable when the amplitude of the sinusoidal inputs is small.

For simplicity we consider a one-dimensional LIN in which lateral connections depend on the spatial separation of the pair of neurons concerned. We also neglect edge effects by taking the network to be of infinite extent. (Note that the one-dimensional case is appropriate when studying the auditory system where at least in the more peripheral regions one has a one-dimensional tonotopic map [16]. One could easily extend the analysis to the two-dimensional case as needed in the retinal system.) We first assume that the background is uniform across the network (Sec. III). This implies that we can take a Fourier transform with respect to the spatial coordinate thus transforming the network into an (infinite) set of in- 
dependent leaky-integrator neurons labeled by the spatial frequency. The effect of the background on each of these can be analyzed as before. However, we find an important difference between recurrent and nonrecurrent LIN topologies $[12,16]$. In the latter case the spatial and temporal components of network response are decoupled so that in particular the effective membrane time constant arising from the background is independent of the spatial frequency. This is no longer true for the recurrent network where spatial and temporal components are strongly coupled. We investigate how the random background affects the high-pass spatial filtering properties of lateral inhibitory networks, particularly those with recurrent topologies.

Finally, we consider a nonuniform background in which each neuron is modulated by an independent colored noise process (Sec. IV). The linear response function of the network now takes the form of a time-ordered exponential matrix operator. This is formally similar to an operator obtained in the analysis of excitons moving along a one-dimensional lattice with random modulations of the local energy at each site [19,7]. Exploiting this similarity, we use a dynamical version of the coherent potential approximation [19] to reduce the network with nonuniform stochastic background to one with an effective uniform time-independent background. This results in a self-consistency condition for the effective background that involves the average response function of a LIN with random fluctuations at a single node of the network (single-site dynamical disorder). This response function is calculated using an extension of the method of partial cumulants applied to the single-neuron model.

\section{LEAKY-INTEGRATOR NEURON IN A STOCHASTIC BACKGROUND}

The dynamics of a leaky-integrator neuron may be described by the equation

$$
\begin{aligned}
\frac{d}{d t} V(t)= & -\frac{V(t)}{\tau}+E(t)\left[S^{(e)}-V(t)\right] \\
& +I(t)\left[S^{(i)}-V(t)\right],
\end{aligned}
$$

where $\tau$ is the membrane time constant, $V$ is the (somatic) membrane potential, $E$ and $I$ are the rates of excitatory and inhibitory input stimulation, and $S^{(e)}, S^{(i)}$ are the associated membrane reversal potentials with $S^{(e)}>0$, $S^{(i)} \leq 0$. Note that $\tau=R C$ where $R$ and $C$ are, respectively, the leakage resistance and capacitance of the neuron. It follows immediately from Eq. (2.1) that both excitatory and inhibitory inputs reduce the effective membrane time constant according to $\tau^{-1} \rightarrow \tau^{-1}+E(t)+l(t)$. (More precisely, the conductance changes induced by synaptic inputs reduce the effective leakage resistance $R$ and hence $\tau$.) A well-known consequence of this so-called shunting effect is that it leads to a compression of the dynamical range of a neuron. To illustrate this, assume that $E$ and $I$ are time independent; the steady-state membrane potential is then

$$
V^{*}=\frac{E S^{(e)}+I S^{(i)}}{\tau^{-1}+E+I}
$$

Thus, rather than $V^{*}$ increasing linearly with $E$ (for fixed $I$, one finds that $V^{*} \rightarrow S^{(e)}$ as $E \rightarrow \infty$. The nonlinear relationship in Eq. (2.2) helps to explain the Weber-Fechner law of retinal processing [20]. Moreover, its generalization to the case of neurons with dendritic structure provides a mechanism whereby recurrent networks can sustain states of firing well below the maximum possible firing rate of a neuron $[4,21]$.

Equation (2.2) also illustrates another important feature. Suppose that $I$ is taken to represent background shunting inhibition with $S^{(i)}=0$. Then $V^{*}=E S^{(e)} /\left(E+I+\tau^{-1}\right)$, i.e., the background does not directly contribute to the input stimulation of the neuron, but does affect the neuron's behavior indirectly by reducing the membrane time constant. In this paper, we shall analyze the consequences of such an effect when there exists a fluctuating background. As a first step, consider a leaky-integrator neuron satisfying

$$
\frac{d V}{d t}=-\frac{V(t)}{\tau}+X(t)-\left[\xi_{0}+\xi(t)\right] V(t),
$$

where $X(t)$ is the net input stimulation of the neuron and the last term on the right-hand side represents background synaptic activity in the form of shunting inhibition. The background is decomposed into a constant component $\xi_{0}$ and a stochastic (zero mean) component $\xi(t)$. The latter is taken to be a multicomponent dichotomous colored noise process. That is, $\xi(t)=\sum_{\mu=1}^{M} \xi^{(\mu)}(t)$ where each $\xi^{(\mu)}(t)$ is a stationary dichotomous colored noise process and the composed process is determined completely in terms of the first and second moments

$$
\begin{aligned}
& \left\langle\xi^{(\mu)}(t)\right\rangle=0, \\
& \left\langle\xi^{(\mu)}(t) \xi^{(v)}\left(t^{\prime}\right)\right\rangle=\delta_{\mu \nu} \gamma^{2} \exp \left(-\lambda\left|t-t^{\prime}\right|\right) .
\end{aligned}
$$

Here \langle\rangle denotes averaging over the stochastic process, $\gamma$ is the strength of the background fluctuations, and $\lambda^{-1}$ is the correlation time. One can view the composed process $\xi(t)$ as jumping between the values $\pm \gamma \pm, \gamma \pm \cdots \pm \gamma(M$ terms) so reflecting different levels of background activity. Since the rate of inhibition is a positive number, we require that $\xi_{0}+\xi(t) \geq 0$. This leads to the condition $M \gamma \leq \xi_{0}$. As a final simplification, we assume that the stochastic background is independent of the input $X(t)$.

Equation (2.3) is an example of a stochastic differential equation with multiplicative colored noise. A full analysis of such equations is generally not possible and one usually has to resort to taking a white-noise limit, for example, so that a Fokker-Planck equation can be constructed. Here, however, we are only concerned with determining the average response of the neuron; this can be calculated exactly. Thus we imagine that there is an ensemble or population of $N$ identical, independent neurons operating in approximately a linear regime so that the average output of the population is $N\langle V(t)\rangle$. First, we integrate Eq. (2.3) under the initial condition $V(0)=0$ and average over the stochastic process. The result is

$$
\langle V(t)\rangle=\int_{0}^{t} H\left(t^{\prime}\right) X\left(t-t^{\prime}\right) d t^{\prime},
$$

where 


$$
H(t)=\exp (-\epsilon t)\left\langle\exp \left[-\int_{0}^{t} \xi\left(t^{\prime}\right) d t^{\prime}\right]\right\rangle
$$

and $\epsilon=\tau^{-1}+\xi_{0}$. Taking the Laplace transform of Eq. (2.5) gives

$$
v(z)=h(z) x(z),
$$

where $h(z), v(z)$, and $x(z)$ are, respectively, the Laplace transform of $H(t),\langle V(t)\rangle$, and the input $X(t)$. For constant inputs, $X(t)=X_{0}$, the average steady state is $h(0) X_{0}$. On the other hand, for sinusoidal inputs of angular frequency $\omega$ the associated transfer function is $h(i \omega)$.

The average response function $H(t)$ is formally similar to the so-called relaxation function of a randomly modulated oscillator [6]. The latter is one of the simplest examples of a physical system interacting with a heat bath. Once the formal connection between a neuron with random synaptic background activity and a system in a heat bath has been made, it follows that the various techniques used to study the latter can also be applied to the former with only minor alterations. (Of course the interpretation of the results will differ considerably in the two cases.) In particular we can calculate $h(z)$ using the timeconvolution generalized master equation (TC-GME) approach [9] combined with the method of partial cumulants [10].

Consider the stochastic differential equation

$$
\frac{d G}{d t}=-[\epsilon+\xi(t)] G(t), \quad G(0)=0 .
$$

We proceed by averaging Eq. (2.8) over the stochastic process without first solving it; this naturally leads to the partial cumulants, which can be calculated explicitly. To achieve the averaging procedure, it is convenient to introduce the projection operator $\mathcal{P}$, which averages everything to the right of it and to let $\mathcal{L}=1-\mathcal{P}$. By definition $H(t)=\mathscr{P} G(t)$. The vanishing of the first moment in Eq. (2.4) implies that $\mathcal{P} \xi(t) \mathscr{P}=0$. Acting from the right on both sides of Eq. (2.8) with the operator $\mathcal{P}$ or $\mathcal{L}$, we obtain the two equations

$$
\frac{d}{d t} \mathcal{P} G(t)=-\epsilon \mathscr{P} G(t)-\mathscr{P} \xi(t) \mathcal{L} G(t)
$$

and

$$
\frac{d}{d t} \mathcal{L} G(t)=-\{\epsilon+\mathcal{L} \xi(t)\} \mathcal{L} G(t)-\xi(t) \mathcal{P} G(t) .
$$

Solving for the quantity $\mathcal{L} G(t)$ by integrating Eq. (2.10) and using $G(0)=0$, we obtain the integro-differential equation (scalar TC-GME),

$$
\frac{d}{d t} H(t)=-\epsilon H(t)+\int_{0}^{t} K\left(t-t^{\prime}\right) H\left(t^{\prime}\right) d t^{\prime},
$$

where $K$ is the so-called memory operator

$$
K(t)=\mathcal{P} \xi(t) \exp \left(-\int_{0}^{t}\left[\epsilon+\mathcal{L} \xi\left(t^{\prime}\right)\right] d t^{\prime}\right) \xi(0), .
$$

Expanding the exponential in Eq. (2.12), which effectively corresponds to a power series expansion in the background strength $\gamma$, we obtain the result
$K(t)=\sum_{n=1}^{\infty} \int_{0}^{t} d t_{2 n-2} \ldots \int_{0}^{t_{2}} d t_{1} c^{(2 n)}\left(0, t_{1}, \ldots, t_{2 n-2}, t\right)$

where the $c^{(2 n)}\left(0, t_{1}, \ldots, t\right), n=1,2, \ldots$ are the partial cumulants for the stochastic process $\xi(t)$,

$$
\begin{aligned}
c^{(2 n)}\left(0, t_{1}, \ldots, t_{2 n-2}, t\right) \\
\quad=\mathscr{P} \xi(t) \mathcal{L} \xi\left(t_{2 n-2}\right) \mathcal{L} \cdots \mathcal{L} \xi\left(t_{1}\right) \mathcal{L} \xi(0) .
\end{aligned}
$$

Note that all partial cumulants of odd order are zero due to the fact that the stochastic process has zero mean.

The partial cumulants for dichotomous colored noise are calculated in Ref. [11] using diagrammatic techniques. It turns out that $c^{(2 n)}$ displays a multiconvolution form involving the temporal differences $\tau_{i}=t_{i}-t_{i-1}, i=1, \ldots, 2 n-1 \quad$ (with $\quad t_{0}=0, t_{2 n-1}=t$ ). Therefore, we can Laplace transform Eq. (2.13) and after some rearrangement express the Laplace transform $k(z)$ of $K(t)$ as a continued fraction

$$
k(z)=\frac{\gamma^{2} \theta_{1}^{M}}{z+\epsilon+\lambda-\frac{\gamma^{2} \theta_{2}^{M}}{z+\epsilon+2 \lambda-\frac{\gamma^{2} \theta_{3}^{M}}{\ddots-\gamma^{2} \theta_{M}^{M}(z+\epsilon+M \lambda)}},}
$$

where $\lambda^{-1}$ is the correlation time and $\theta_{k}^{M}=k(M+1-k)$. Note that the continued fraction can be rewritten in the standard form

$$
\frac{1}{a_{1}+\frac{1}{a_{2}+\frac{1}{\ddots+\frac{1}{a_{M}}}}},
$$

where

$a_{k}=b_{k}(z+\epsilon+k \lambda), \quad b_{k}=-\frac{1}{\theta_{k}^{M} \gamma^{2} b_{k-1}}, \quad b_{1}=\frac{1}{\gamma^{2} \theta_{1}^{M}}$.

It is clear that $\left\{b_{1}, b_{2}, \ldots, b_{M}\right\}$ forms an alternating sequence. This differs from the analogous expression for the randomly modulated oscillator where the $b_{k}$ are all positive. The presence of an alternating sequence means that $k(z)$ can have singularities when $z$ is real. However, these singularities will be absent in our model provided the $\operatorname{Re} z>-\xi_{0}$, which reflects the dissipative nature of the underlying neuron model. This also implies that for real $z, k(z)>0$ if $z>-\xi_{0} ; k(z)$ is then a monotonically decreasing function of $z$.

Having obtained the Laplace transform of the memory function, we can now proceed to solve Eq. (2.11). Laplace transforming both sides of Eq. (2.11) gives

$$
h(z)=\frac{1}{z+\epsilon-k(z)} .
$$


First, consider the case of a constant input $X(t)=X_{0}$. Setting $z=0$ in Eq. (2.18), it can be seen that the net effect of the background is to modify the membrane time constant according to

$$
\frac{1}{\tau} \rightarrow \frac{1}{\tau}+\xi_{0}-k(0) \text {. }
$$

Thus averaging over the stochastic background contribution $\xi(t)$ results in a smaller reduction in $\tau$ than a constant background of the same average intensity. Also note that $k(0)$ increases with the order $M$ of the dichotomous noise process provided that the condition $M \gamma<\xi_{0}$ is not violated.

Next consider the response to sinusoidal inputs. Averaging over the fluctuating background now results in a frequency-dependent contribution to the average transfer function, which leads to nontrivial modifications in both the phase and amplitude of response. This is illustrated in Figs. 1 and 2 where we have plotted $P(\omega)=|h(i \omega)|^{2}$ and $\theta(\omega)=\arg h(i \omega)$ against frequency $\omega$ for single component dichotomous noise. Setting $M=1$ in Eq. (2.15) gives the transfer function

$$
h(i \omega)=\frac{1}{\epsilon+i \omega-\gamma^{2}(\epsilon+i \omega+\lambda)^{-1}} .
$$

We consider the two cases (a) $\gamma=0$ (zero fluctuations) and (b) $\gamma=0.7$. We also set $\epsilon=1$ and $\lambda=0$ (static limit). It can be seen from Fig. 1 that the leaky-integrator neuron acts like a low-pass filter. The random background modifies the response in two ways. First, the maximum response (at $\omega=0$ ) increases with $\gamma$ as a consequence of the transformation (2.19). Second, the frequency dependence of the effective background leads to a narrowing of the response curve as can be seen by comparing curve (b) in Fig. 1 with the dashed curve; the latter describes the response of the neuron with frequency-independent background activity $\xi_{0}-k(0)$. The combination of these two features gives a more sharply tuned low-pass filter. Similar conclusions can be drawn from the phase plot of Fig. 2. Similar behavior to Figs. 1 and 2 occurs for multicomponent dichotomous noise $(M>1)$. Finally, as one might expect, the influence of the fluctuating background $\xi(t)$

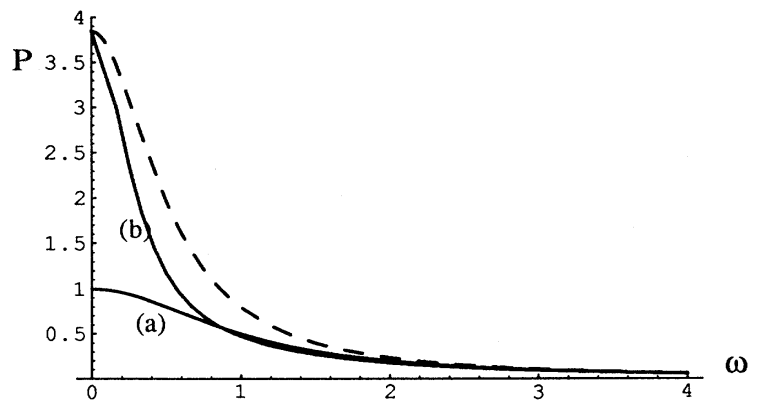

FIG. 1. Average response of a leaky-integrator neuron in a stochastic background: plot of intensity $P(\omega)$ against frequency $\omega$ for (a) $\gamma=0$ (zero background) and (b) $\gamma=0.7$. The dashed curve shows the response due to a frequency-independent background contribution $\xi_{0}-k(0)$.

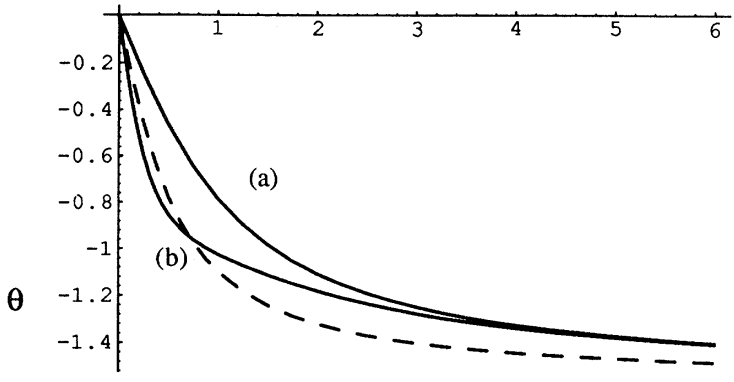

$\omega$

FIG. 2. Average response of a leaky-integrator neuron in a stochastic background: plot of phase $\theta(\omega)$ against frequency $\omega$ for (a) $\gamma=0$ and (b) $\gamma=0.7$. The dashed curve constructed as in Fig. 1.

decreases as the correlation time decreases. In the limit $\lambda \rightarrow 0$ the noise process becomes completely incoherent and gives zero contribution to the average response.

\section{LATERAL INHIBITORY NETWORKS IN A UNIFORM BACKGROUND}

As discussed by Hartline [12] and Shamma [16], there are two types of topological arrangement for LIN's. These are illustrated in Figs. 3 and 4 for a onedimensional network. For simplicity we shall consider linear models for which the output firing rate $f$ of each neuron is of the form $f(t)=\kappa V(t)$, where $\kappa$ is some constant. In the case of a nonrecurrent network, the input $X_{n}(t)$ to the $n$th neuron inhibits the other neurons $m \neq n$ along weights $W_{\mathrm{mn}}$. Thus

$$
\frac{d V_{n}}{d t}=-\frac{V_{n}(t)}{\tau}+X_{n}(t)-\sum_{m \neq n} W_{n m} X_{m}(t),
$$

where $V_{n}(t)$ is the membrane potential of the $n$th neuron and $\tau=R C$ with the membrane resistance and capacitance assumed to be $n$ independent. [Note that $C X_{n}(t)$ has the units of current.] On the other hand, a recurrent network employs inhibitory feedback,

$$
\frac{d V_{n}}{d t}=-\frac{V_{n}(t)}{\tau}-\sum_{m \neq n} W_{n m} V_{m}(t)+X_{n}(t)
$$

In Eq. (3.2), the factor $\kappa$ relating membrane potential to

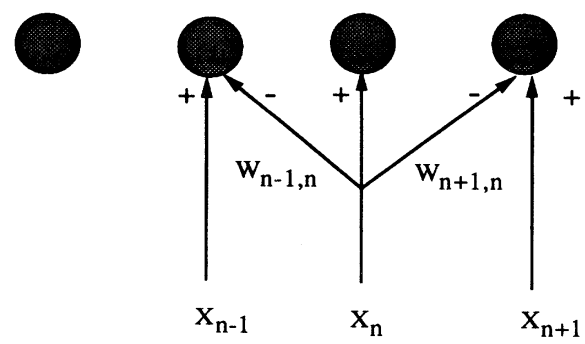

FIG. 3. Nonrecurrent lateral inhibitory network. 
firing rate has been absorbed into the weights $W_{n m}$. We assume that for both topologies the weights $W_{m n}$ depend on the distance of separation between the pair $(m n)$ and are symmetric. In other words,

$$
W_{m m}=W(m-n)=W(n-m) .
$$

The network is also taken to be of infinite extent so that end effects can be ignored. One subtle difference between the two types of network concerns the units of the weights $W_{m n}$. That is, $W_{n m}$ is dimensionless for the nonrecurrent topology whereas it has the units of $\sec ^{-1}$ in the recurrent case.

Fourier transforming Eqs. (3.1) and (3.2) with respect to the spatial coordinate gives

$$
\frac{d V(p, t)}{d t}=-\left(\frac{1}{\tau}+W(p)\right) V(p, t)+X(p, t), \text { recurrent },
$$

$$
\frac{d V(p, t)}{d t}=-\frac{1}{\tau} V(p, t)+[1-W(p)] X(p, t),
$$

nonrecurrent

with

$$
V(p)=\sum_{-\infty}^{\infty} e^{i p n} V_{n}, \quad W(p)=\sum_{-\infty}^{\infty} e^{i p n} W(n) .
$$

Thus the network reduces to an (infinite) set of independent leaky-integrator neurons labeled by the spatial frequency $p$. The transfer function of the network is obtained by Laplace transforming Eqs. (3.4) and (3.5) with respect to time $t$,

$$
\begin{aligned}
& h_{\mathrm{R}}(z, p)=\frac{1}{z+\tau^{-1}+W(p)}, \\
& h_{\mathrm{NR}}(z, p)=\frac{1-W(p)}{z+\tau^{-1}} .
\end{aligned}
$$

If temporal variations in the input are slow relative to the time constant $\tau$ then the temporal dependence of the LIN output can be approximately ignored; to a first approximation one can set $z=0$ in Eqs. (3.7) and (3.8). Based on physiological findings [12], the weight distribution $W(n)$ can be taken as a slowly decreasing function of $n$. We shall assume for concreteness that the Fourier transform

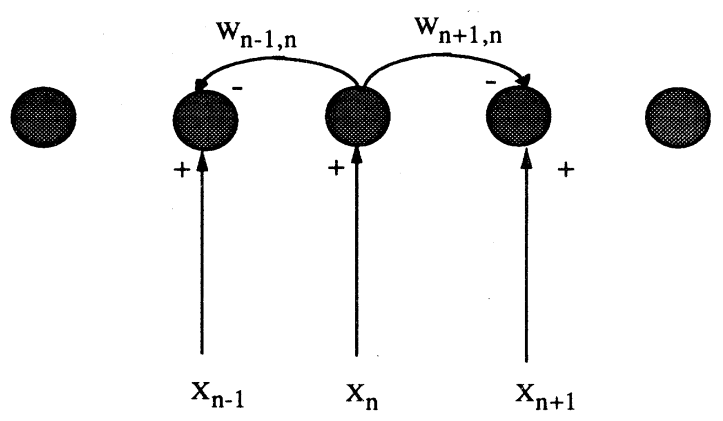

FIG. 4. Recurrent lateral inhibitory network. is $W(p)=W_{0} \exp \left(-p^{2}\right)$. This produces for a recurrent network a transfer function $h_{\mathrm{R}}(0, p)$ of the form illustrated in Fig. 5 for $W_{0}=0.5, \tau=1$. We see that the network performs a spatial high-pass filtering operation on the input; this corresponds functionally to the sharpening of the steady-state input patterns as mentioned in the Introduction. Similar behavior is exhibited by the nonrecurrent architecture.

However, differences emerge between the two types of LIN in the presence of temporal variations of the input. In the nonrecurrent case, temporal and spatial components are decoupled with the former consisting of a low-pass filter. That is, patterns oscillating at high temporal frequencies $(\gg 1 / \tau)$ are severely attenuated. On the other hand, there is strong coupling between temporal and spatial components in the recurrent LIN as can be seen by rewriting Eq. (3.7) as

$h_{\mathrm{R}}(i \omega, p)=\frac{1}{i \omega \tau_{\mathrm{eff}}+1} \frac{\tau}{1+\tau W(p)}, \quad \tau_{\mathrm{eff}}=\frac{\tau}{1+\tau W(p)}$.

The effective time constant of the low-pass filter $\tau_{\text {eff }}$ depends on $W(p)$. For higher spatial frequencies, $W(p) \rightarrow 0$, so that the time constant $\tau_{\text {eff }}$ increases and there is a greater attenuation of high temporal frequency oscillations.

The above difference between recurrent and nonrecurrent networks is important when one introduces stochastic background activity along the lines of Sec. II. Assume for the moment that the background is spatially uniform. (See Sec. IV for an analysis of a nonuniform background.) That is, an additional term is introduced on the right-hand side of Eqs. (3.1) and (3.2) given by $-\left[\xi_{0}+\xi(t)\right] V_{m}(t)$. Since the background is uniform we can still take the spatial Fourier transform. This reduces the LIN an equivalent set of independent leaky-integrator neurons each of which is in a stochastic background. Since the spatial and temporal parts are decoupled in the nonrecurrent case, the analysis of Sec. II gives the result that the average transfer function is

$$
h_{\mathrm{NR}}(z, p)=\frac{1-W(p)}{z+\epsilon-k(z)} .
$$

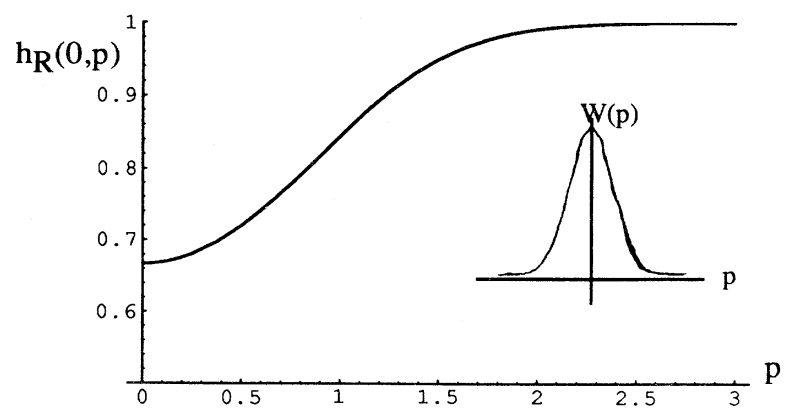

FIG. 5. Spatial transfer function $h_{\mathrm{R}}(0, p)$ against frequency $p$ for a recurrent LIN with zero background fluctuations. The inset is the Fourier transform of the inhibitory feedback weight distribution. 
The contribution $k(z)$ from the stochastic background is $p$ independent. On the other hand, the average transfer function for a recurrent LIN is

$$
h_{\mathrm{R}}(z, p)=\frac{1}{z+\epsilon+W(p)-k(z+W(p))},
$$

where $k(z)$ is defined by Eq. (2.15).

The spatial transfer function $h_{\mathrm{R}}(0, p)$ of a recurrent LIN is plotted in Fig. 6 for a range of background intensities $\gamma$. We have taken $M=1, \epsilon=1, \lambda=0$, and $W_{0}=0.5$. It can be seen that the fluctuating background leads to an enhancement of the spatial high-pass filtering properties of the network. Comparing the high- and low-frequency response using Eqs. (3.11) and (2.15) shows that for $M=1$

$$
\begin{aligned}
& h_{\mathrm{R}}(0,0)=\frac{1}{\epsilon+W_{0}-\gamma^{2} /\left(\epsilon+W_{0}\right)}, \\
& h_{\mathrm{R}}(0, \infty)=\frac{1}{\epsilon-\gamma^{2} / \epsilon} .
\end{aligned}
$$

If $W_{0}$ is sufficiently large, the $\gamma$-dependent correction to the low-frequency response is small, $h_{\mathrm{R}}(0,0) \approx(\epsilon$ $\left.+W_{0}\right)^{-1}$ for all $\gamma$. On the other hand, the highfrequency response is greatly enhanced as $\gamma$ increases from zero. This degree of enhancement would not be obtained by changes in the level of constant background activity $\xi_{0}$. [There would also need to occur changes in the weight distribution $W(p)$.] Similar behavior is found in the case of a nonrecurrent LIN. Equation (3.10) implies that $h_{\mathrm{NR}}(0, \infty)=h_{\mathrm{R}}(0, \infty)$. If $\epsilon+W_{0}<1$ then $h_{\mathrm{NR}}(0,0)$ $>h_{\mathrm{R}}(0,0)$ for all $\gamma$. If $\epsilon+W_{0}>1$ then $h_{\mathrm{NR}}(0,0)$ $<h_{\mathrm{R}}(0,0)$ for $\gamma=0$ and there exists a critical value $\gamma_{c}$ such that $h_{\mathrm{NR}}(0, \infty)>h_{\mathrm{R}}(0, \infty)$ for $\gamma>\gamma_{c}$ where

$$
\gamma_{c}^{2}=\frac{\epsilon\left(\epsilon+W_{0}\right) W_{0}\left(\epsilon+W_{0}-1\right)}{W_{0}(\epsilon+1)} .
$$

Thus for large values of $\gamma$ the degree of contrast between high- and low-frequency response can be considerably smaller for a nonrecurrent network. This is illustrated in Fig. 7 for the same parameter values as used in Fig. 6.

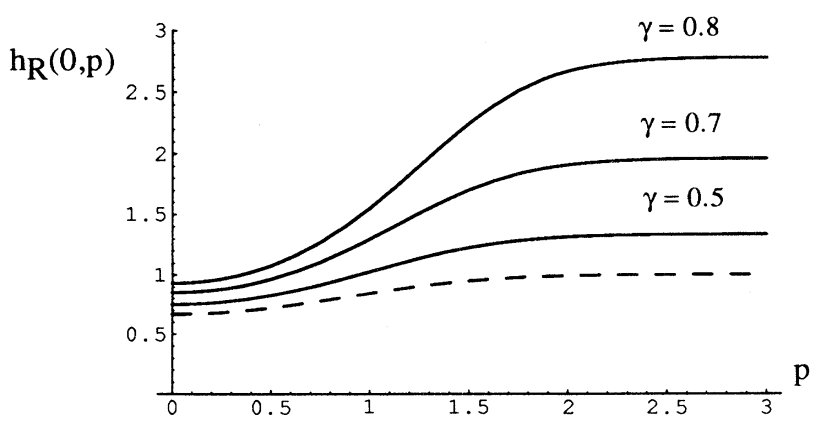

FIG. 6. Spatial transfer function $h_{\mathrm{R}}(0, p)$ of a recurrent LIN in a uniform background for a range of values of background intensity $\gamma$. The dashed curve corresponds to zero background $(\gamma=0)$.

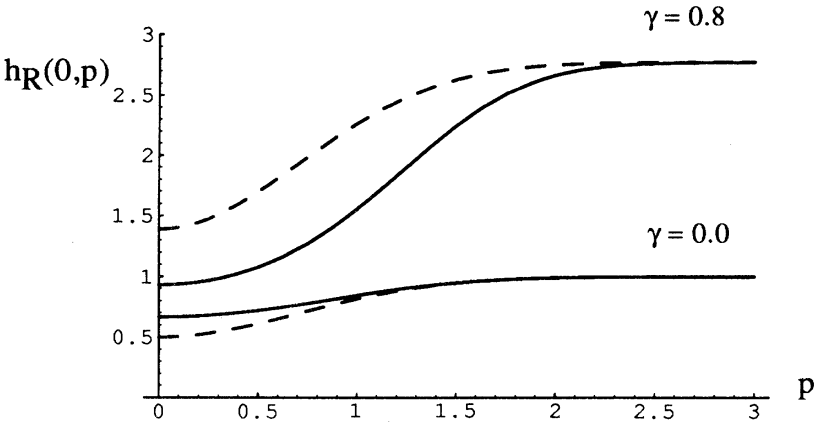

FIG. 7. Comparison of spatial frequency response of recurrent (solid curve) and nonrecurrent (dashed curve) LIN's for (a) $\gamma=0.0$ and (b) $\gamma=0.8$.

\section{LATERAL INHIBITORY NETWORK IN A NONUNIFORM BACKGROUND}

Suppose that each neuron $n$ of a recurrent LIN independently receives synaptic background activity such that

$$
\begin{aligned}
\frac{d V_{n}}{d t}= & -\frac{V_{n}}{\tau}-\sum_{m \neq n} W_{m n} V_{n}(t)+X_{n}(t) \\
& -\left[\xi_{0}+\xi_{n}(t)\right] V_{n}(t)
\end{aligned}
$$

where each $\xi_{n}(t)$ is a multicomponent dichotomous colored noise process with

$$
\begin{aligned}
& \left\langle\xi_{n}^{(\mu)}(t)\right\rangle=0, \\
& \left\langle\xi_{m}^{(\mu)}(t) \xi_{n}^{(v)}\left(t^{\prime}\right)\right\rangle=\delta_{m n} \delta^{\mu v} \gamma^{2} \exp \left(-\lambda\left|t-t^{\prime}\right|\right)
\end{aligned}
$$

and the weights $W_{m n}$ satisfy Eq. (3.3). In contrast with the uniform background case, it is no longer possible to Fourier transform Eq. (4.1) directly to obtain a singleneuron model; this is due to the presence of the term $-\xi_{m}(t) V_{m}(t)$. Formally integrating Eq. (4.1) gives [with $V_{m}(0)=0$ for all $\left.m\right]$

$$
V_{m}(t)=\int_{0}^{t} \sum_{n} G_{m n}\left(t, t^{\prime}\right) X_{n}\left(t^{\prime}\right) d t^{\prime},
$$

where the response matrix $\mathbf{G}$ satisfies

$$
\frac{d}{d t} \mathbf{G}(t, 0)=-\mathbf{Q}(t) \mathbf{G}(t, 0), \mathbf{G}(0,0)=1 \text {. }
$$

Here

$$
\begin{aligned}
& \mathbf{Q}(t)=\mathbf{Q}^{(0)}+\mathbf{Q}^{(1)}(t), \quad Q_{m n}^{(0)}=\epsilon \delta_{m n}+W_{m n}, \\
& Q_{m m}^{(1)}(t)=\xi_{m}(t) \delta_{m n} .
\end{aligned}
$$

Solving Eq. (4.4),

$$
\mathbf{G}\left(t, t^{\prime}\right)=\mathbf{T}\left[\exp \left[-\int_{t^{\prime}}^{t} \mathbf{Q}\left(t^{\prime \prime}\right) d t^{\prime \prime}\right]\right] \text {, }
$$

where $\mathbf{T}$ is the time-ordering operator. Note that the response matrix $\mathbf{G}\left(t, t^{\prime}\right)$ does not simply depend on the 
time difference $t-t^{\prime}$. However, time-translation invariance is recovered on averaging over the stochastic processes $\xi_{m}(t)$ since the latter are generated by a stationary process. Thus we can define an averaged response matrix H such that

$$
\mathbf{H}\left(t-t^{\prime}\right)=\left\langle\mathbf{G}\left(t, t^{\prime}\right)\right\rangle .
$$

This in turn implies that after averaging Eq. (4.3) one can take the Laplace transform to obtain the result

$$
v_{m}(z)=\sum_{n} h_{m n}(z) x_{n}(z)
$$

The averaged LIN response matrix $\mathbf{H}(t)$ defined by Eqs. (4.6) and (4.7) is formally identical to the propagator describing excitons moving along a one-dimensional lattice with random modulations of the local energy at each lattice site [19]. (These modulations represent the effects of a heat bath.) Therefore, as in Sec. II, we can use techniques from nonequilibrium statistical physics to evaluate $\mathbf{H}(t)$. In particular, following Sumi [19] we use a dynamical coherent potential approximation to replace the random fluctuations $\xi_{n}(t)$ appearing in the matrix $Q(t)$ of Eq. (4.6) by an effective site-independent constant synaptic background $\Lambda$ so that

$\mathbf{H}(t) \approx \hat{\mathbf{G}}(t)=\exp (-t \hat{\mathbf{Q}}), \quad \widehat{\mathbf{Q}}=\mathbf{Q}^{(0)}+\Lambda \mathbf{1}$.

The self-energy term $\Lambda$ is determined by a selfconsistency condition, which ensures that any statistical fluctuations at the single-site level are taken into account.

The derivation of the self-consistency condition for $\Lambda$ proceeds along analogous lines to [19]. First, write the matrix $\mathbf{Q}(t)$ as

$$
Q_{m n}(t)=[\hat{Q}]_{m n}+\left[\xi_{m}(t)-\Lambda\right] \delta_{m n} .
$$

The background $\Lambda$ is determined by the condition that statistical fluctuations arising from the second term on the right-hand side vanish at each single site. (Under the coherent potential approximation one neglects multisite correlations.) It then suffices to consider a LIN in which temporal fluctuations occur at only one site or neuron, $n=0$, say. The corresponding matrix $\widetilde{\mathbf{Q}}$ is

$$
\begin{aligned}
\widetilde{Q}_{m n}(t) & =\widehat{Q}_{m n}+\left[\xi_{0}(t)-\Lambda\right] \delta_{m 0} \delta_{n 0} \\
& =\widetilde{Q}_{m n}^{(0)}+\widetilde{Q}_{m n}^{(1)}(t)
\end{aligned}
$$

with

$\widetilde{Q}_{m n}^{(0)}=\widehat{Q}_{m n}-\Lambda \delta_{m 0} \delta_{n 0}, \quad \widetilde{Q}^{(1)}(t)=\xi_{0}(t) \delta_{m 0} \delta_{n 0}$.

Introducing the modified average response matrix

$$
\widetilde{\mathbf{H}}(t)=\left\langle\mathbf{T}\left[\exp \left[-\int_{0}^{t} \widetilde{\mathbf{Q}}\left(t^{\prime}\right) d t^{\prime}\right]\right]\right\rangle
$$

the self-consistency conditions for $\Lambda$ takes the form [19]

$$
\widetilde{H}_{00}(t)=\hat{G}_{00}(t) \text {. }
$$

In order to determine the effective background $\Lambda$, we still have to calculate the modified matrix $\widetilde{\mathbf{H}}(t)$ which describes the average response of a LIN with single-site dynamical disorder. The latter can be achieved using an extension of the method of partial cumulants detailed in Sec. II. First, we average the matrix Eq. (4.4) with $Q$ replaced by $\widetilde{\mathbf{Q}}$ using the operators $\mathcal{P}$ and $\mathcal{L}$. This gives the two matrix equations

$\frac{d}{d t} \mathcal{P} \mathbf{G}(t, 0)=-\widetilde{\mathbf{Q}}^{(0)} \mathcal{P} \mathbf{G}(t, 0)-\mathcal{P} \widetilde{\mathbf{Q}}^{(1)}(t) \mathcal{L} \mathbf{G}(t, 0)$

and

$$
\begin{aligned}
\frac{d}{d t} \mathcal{L} \mathbf{G}(t, 0)= & -\left\{\widetilde{\mathbf{Q}}^{(0)}+\mathcal{L} \widetilde{\mathbf{Q}}^{(1)}(t)\right\} \mathcal{L} \mathbf{G}(t, 0) \\
& -\widetilde{\mathbf{Q}}^{(1)}(t) \mathcal{P} \mathbf{G}(t, 0) .
\end{aligned}
$$

Solving for the quantity $\mathcal{L} \mathbf{G}(t, 0)$ by integrating Eq. (4.16) and using the identity $\mathbf{G}(0,0)=1$, we obtain the TCGME

$\frac{d}{d t} \widetilde{\mathbf{H}}(t)=-\widetilde{\mathbf{Q}}^{(0)} \widetilde{\mathbf{H}}(t)+\int_{0}^{t} \mathbf{K}\left(t-t^{\prime}\right) \widetilde{\mathbf{H}}\left(t^{\prime}\right) d t^{\prime}$,

where the memory operator $\mathbf{K}$ satisfies

$$
\begin{aligned}
\mathbf{K}\left(t-t^{\prime}\right)= & \mathcal{P} \widetilde{\mathbf{Q}}^{(1)}(t) \mathbf{T}\left[\exp \left[-\int_{t^{\prime}}^{t}\left[\widetilde{\mathbf{Q}}^{(0)}+\mathcal{L} \widetilde{\mathbf{Q}}^{(1)}\left(t^{\prime}\right)\right] d t^{\prime}\right]\right] \\
& \times \widetilde{\mathbf{Q}}^{(1)}\left(t^{\prime}\right) .
\end{aligned}
$$

Since the random background is now assumed to impinge on a single neuron alone (single-site modulation), one finds that the memory operator matrix $\mathbf{K}(t)$ has a single nonzero matrix element, that is, $K_{m n}(t)=K(t) \delta_{m, 0} \delta_{n, 0}$. As in the scalar example of Sec. II, $K(t)$ may be expanded in terms of the partial cumulants (2.14)

$$
\begin{gathered}
K(t)=\sum_{n=1}^{\infty} \int_{0}^{t} d t_{2 n-2} \ldots \int_{0}^{t_{2}} d t_{1} c^{(2 n)}\left(0, t_{1}, \ldots, t_{2 n-2}, t\right) \\
\times \prod_{i=1}^{2 n-1} \widetilde{G}_{00}\left(\tau_{i}\right)
\end{gathered}
$$

where

$$
\widetilde{\mathbf{G}}(t)=\exp \left(-t \widetilde{\mathbf{Q}}^{(0)}\right) .
$$

Since the $c^{(2 n)}$ depend on the same set of time differences $\tau_{i}$ as appears in the product on the right-hand side of Eq. (4.19), the individual terms of the expansion (4.19) display a multiconvolution form. Therefore, we can Laplace transform Eq. (4.19) and after some rearrangement express $k(z)$ as a continued fraction identical in form to Eq. (3.12)

$$
k(z)=\frac{\gamma^{2} \theta_{1}^{M}}{g(z+\lambda)^{-1}-\frac{\gamma^{2} \theta_{2}^{M}}{g(z+2 \lambda)^{-1}-\frac{\gamma^{2} \theta_{3}^{M}}{\ddots-\frac{\gamma^{2} \theta_{M}^{M}}{g(z+M \lambda)^{-1}}}}}
$$

with

$g(z) \equiv \widetilde{g}_{00}(z)=\left[\widehat{g}_{00}(z)^{-1}-\Lambda\right]^{-1}, \quad \hat{g}(z) \equiv \hat{g}_{00}(z)$ 
and $\widetilde{\mathbf{g}}(z)$ is the Laplace transform of $\widetilde{\mathbf{G}}(t)$, etc. The matrix $\hat{\mathbf{g}}(z)$ can be calculated explicitly by noting that $\hat{\mathbf{G}}(t)$ of Eq. (4.4) is the response matrix of a LIN with siteindependent background $\xi_{0}+\Lambda$. This means that Fourier transforms with respect to spatial location can be taken. The result is

$$
\hat{g}_{m n}(z)=\int_{-\pi}^{\pi} \frac{e^{i p(m-n)}}{z+\epsilon+\Lambda+W(p)} \frac{d p}{2 \pi},
$$

where $W(p)$ is defined by Eq. (3.6).

Having obtained the Laplace transform of the memory operator, we can now proceed to solve Eq. (4.17). Laplace transforming both sides gives

$$
\widetilde{\mathbf{h}}(z)=\left[z 1+\widetilde{\mathbf{Q}}^{(0)}-\mathbf{k}(z)\right]^{-1} .
$$

A series expansion and an application of Dyson's equation then yields

$$
\widetilde{\mathbf{h}}_{m n}(z)=\widetilde{g}_{m n}^{(0)}(z)+\frac{\widetilde{g}_{m 0}^{(0)}(z) k(z) \widetilde{g}_{0 n}^{(0)}(z)}{1-k(z) g(z)} .
$$

In particular, setting $m=n=0$,

$$
\tilde{h}_{00}(z)=\frac{g(z)}{1-k(z) g(z)} .
$$

Combining Eqs. (4.26) and (4.22) with the Laplace transform of (4.14) finally yields the following self-consistency condition for the effective background $\Lambda$ :

$$
\Lambda=-k(z, \Lambda) \text {. }
$$

The dependence of $k(z)$ on the background field through presence of $g(z)$ in Eq. (4.21) has been made explicit.

In conclusion, substituting Eq. (4.9) into (4.8) and Fourier transforming using Eq. (4.23) yields the following result: within a coherent potential approximation the average transfer function of a recurrent LIN with random modulation of each neuron by synaptic background activity is

$$
h_{\mathrm{R}}(z, p)=\frac{1}{z+\epsilon+\Lambda(z)+W(p)},
$$

where $\Lambda(z)$ is the solution to the self-consistency condition (4.27). Comparing with $h_{\mathrm{R}}$ for a uniform background, we see that the effect of the background is $p$ independent; this is to be expected since the background is now spatially incoherent.

We shall now demonstrate that Eq. (4.27) yields a unique nonzero solution for the effective background satisfying $\Lambda(z)<0$. First, consider the case $M=1$. Equations (4.21), (4.22), and (4.27) yield the self-consistency condition

$$
\Lambda=-\gamma^{2} g(z+\lambda)=-\frac{\gamma^{2}}{\hat{g}(z+\lambda)^{-1}-\Lambda} .
$$

This is a quadratic in $\Lambda$ which has the solution

$$
\Lambda=\frac{1}{2}\left[\hat{g}(z+\lambda)^{-1}-\sqrt{\widehat{g}(z+\lambda)^{-2}+4 \gamma^{2}}\right] .
$$

[Only the negative square root is valid since we require $\Lambda(z) \rightarrow 0$ as $\gamma \rightarrow 0$.] Although $\hat{g}(z)$ itself depends on $\Lambda$
[Eq. (4.23)], it is clear from Eq. (4.30) that $\Lambda(z)<0$.

Next consider the case $M=2$. The self-consistency condition now becomes

$$
\Lambda=-\frac{2 \gamma^{2}}{g(z+\lambda)^{-1}-2 \gamma^{2} g(z+2 \lambda)} .
$$

Equation (4.22) and some algebra leads to the equation

$$
\begin{aligned}
& \Lambda[1-\Lambda \hat{g}(z+\lambda)][1-\Lambda \hat{g}(z+2 \lambda)] \\
& =2 \gamma^{2} \hat{g}(z+\lambda)[2 \hat{g}(z+2 \lambda) \Lambda-1] .
\end{aligned}
$$

By continuity from the solution $\Lambda(z)=0$ at $\gamma^{2}=0$, we see that the only physical solution to Eq. (4.32) satisfies $\Lambda(z)<0$. That is, when $\gamma^{2}<<1$ we find that $|\Lambda|<<1$ and hence $\hat{g}(z+\lambda) \Lambda<1,2 \hat{g}(z+2 \lambda) \Lambda<1$. Thus for small $\gamma^{2}$ the term in square brackets on the left-hand side of Eq. (4.32) is positive while the right-hand side is negative, which means that $\Lambda<0$. This solution remains negative for all $\gamma^{2}$. Similarly, one can show that the effective synaptic background is negative for any $M$. However, it should be noted that the physical restriction $M \gamma<\xi_{0}$ implies that $\gamma^{2} \rightarrow 0$ as $M \rightarrow \infty$. Thus within the constraints of the model only low order dichotomous noise process will give a significant effect. In all cases $|\Lambda(z)|$ is a decreasing function of $\lambda$.

A simple approximate expression for the effective synaptic background $\Lambda(z)$ can be obtained when the level of lateral inhibition is small. More specifically, suppose that the distribution of lateral connections is given by $W(p)=W_{0} \exp \left(-p^{2}\right)$ (see Sec. III) such that

$$
\left|\frac{W_{0}}{z+\epsilon+\Lambda}\right|<1 \text {. }
$$

We can then expand the denominator of Eq. (4.23) for $m=n=0$ to obtain

$\hat{\boldsymbol{g}}(z)=\sum_{n=0}^{\infty}(-1)^{n} \frac{\mathcal{J}_{n} W_{0}^{n}}{(z+\epsilon+\Lambda)^{n+1}}$,

where

$\mathcal{J}_{n}=\frac{1}{2 \pi} \int_{-\pi}^{\pi} \exp \left(-n p^{2}\right) d p=\frac{1}{\sqrt{4 \pi n}} \operatorname{erf}(\sqrt{n} \pi)$.

Thus to first order in $W_{0}$,

$$
\hat{g}(z)=\frac{1}{z+\epsilon+\Lambda}\left[1-\frac{\mathcal{J}_{1} W_{0}}{z+\epsilon+\Lambda}\right] .
$$

It follows from Eq. (4.36) that

$$
g(z)^{-1} \approx z+\epsilon+W_{0} \mathcal{J}_{1}
$$

Substituting Eq. (4.37) into Eq. (4.21) shows that the effective background is $\Lambda(z)=-k\left(z+\mathscr{J}_{1} W_{0}\right)$ where $k(z)$ is given by (2.15). [One can check that such a solution is consistent with the condition (4.33) for $W_{0}$ sufficiently small.] Hence, within this approximation the transfer function of a recurrent LIN with nonuniform synaptic background reduces to 


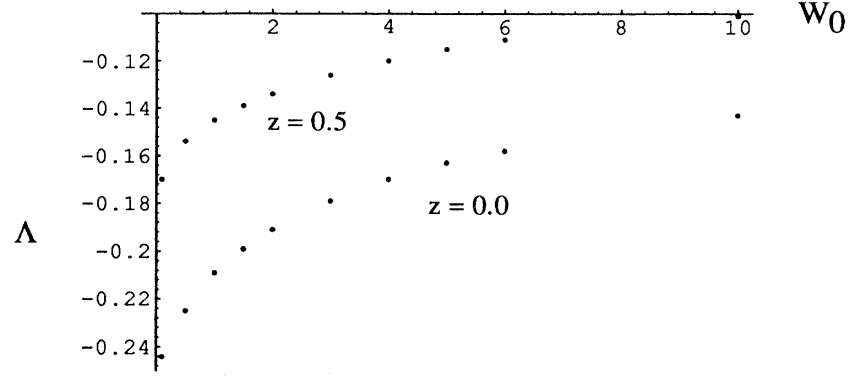

FIG. 8. Variation of effective background $\Lambda(z)$ with amplitude of inhibitory connections $W_{0}$ for $z=0.0$ and $z=0.5$.

$$
\begin{aligned}
& h_{\mathrm{R}}(z, p) \approx \frac{1}{\bar{W}, \tau^{-1}+\xi_{0}+W(p)-k(z+\bar{W})}, \\
& \bar{W}=\frac{1}{2 \pi} \int_{-\pi}^{\pi} W(p) d p .
\end{aligned}
$$

The fact that a nonuniform fluctuating background results in a $p$-independent contribution to the transfer function means that the contrast between high and low spatial frequency responses is not as strong as for a spatially uniform background [see the discussion following Eq. (3.11)]. The above approximation also implies that for small $W_{0}$ and real $z$, the effective background $|\Lambda(z)|$ decreases as $W_{0}$ increases. This in fact holds for all values of $W_{0}$ as is illustrated in Fig. 8 where $\Lambda(z)$ is plotted as a function of $W_{0}$ for $z=0.0$ and $z=0.5$. The effective background $\Lambda(z)$ is obtained by numerically solving Eq. (4.27) for $M=1, \epsilon=1$ and $\lambda=0$.

Finally, note that we have considered two extremes concerning the spatial distribution of background activity across the lateral inhibitory network, namely uniform (Sec. III) and completely random. Although more general spatial distributions are harder to analyze we expect that averaging over the fluctuating background leads to a negative effective background contribution that generally depends on both the spatial and temporal frequency of response. The dependence on spatial frequency will only disappear when spatial correlations of the background activity across the network are absent, as in Sec. IV.

\section{DISCUSSION}

In this paper, we established a formal equivalence between a neuron with random synaptic background activity and a physical system in a heat bath. We then applied techniques from nonequilibrium statistical physics such as the method of partial cumulants and dynamical coherent potential approximation to analyze how a fluc- tuating background influences the spatiotemporal processing properties of lateral inhibitory neural networks. Our main result is that averaging over the background leads to an effective membrane time constant of the system given by

$$
\frac{1}{\tau_{\mathrm{eff}}}=\frac{1}{\tau}+\xi_{0}-\Lambda(\omega, p),
$$

where $\tau$ is the time constant in the absence of any background activity, $\xi_{0}$ is the (constant) mean level of activity, and $\Lambda(\omega, p)$ is the contribution from background fluctuations about the mean with $\Lambda>0$. Note that $\Lambda$ will generally depend on both the spatial frequency $p$ and the temporal frequency $\omega$ of response. Although we restricted ourselves to the case of dichotomous colored noise, we could easily consider a more general process that interpolates between dichotomous, Gaussian, and compound Poisson processes [6].

There are two limitations concerning the present analysis that should be noted. First, the networks were assumed to be linear; although this is a reasonable approximation for small amplitude inputs, it is important to develop techniques for analyzing the effects of background activity on nonlinear networks. For example, a nonlinear extension of Eq. (3.2) is

$$
\begin{aligned}
\frac{d V_{n}}{d t}= & -\frac{V_{n}}{\tau}-\sum_{m \neq n} W_{m n} f\left(V_{m}(t)\right)+x_{n}(t) \\
& +(\text { synaptic background) },
\end{aligned}
$$

where $f$ is a sigmoidal output function of the form $f(x)=[1+\exp (-\kappa x)]^{-1}$. The object of interest is now the average output $\left\langle f\left(V_{m}\right)\right\rangle$. One possible starting point for the analysis of such nonlinear networks is to perform a perturbation expansion in powers of the nonlinear gain $\kappa$. The second limitation of our analysis is that it only determined the average response of the network. Thus one imagines an ensemble of identical networks each with an independent fluctuating background. This raises two important issues: (i) What information is usefully extracted from such an ensemble beyond the average response? (ii) How does one take into account statistical correlations of background activity between elements of the ensemble?

Finally, given that synaptic background activity can influence the behavior of neurons along the lines illustrated in this paper, it is of interest to determine whether or not there is some mechanism for controlling such background activity (by the release of neuromodulators?), and if so, how this mechanism could be exploited by the system. We hope to consider these particular issues elsewhere.
[1] W. R. Holmes and C. D. Woody, Brain Res. 505, 12 (1989).

[2] O. Bernander, R. J. Douglas, A. C. Martin, and C. Koch Proc. Natl. Acad. Sci. USA 88, 11569 (1991).

[3] M. Rapp, Y. Yarom, and I. Segev, Neural Comp. 4, 518 (1992).
[4] P. C. Bressloff and J. G. Taylor, Phys. Rev. E 47, 2899 (1993).

[5] P. C. Bressloff, J. Phys. A 27, 4097 (1994).

[6] R. Kubo, J. Math. Phys. 4, 174 (1963).

[7] P. Chvosta, Z. Phys. B 86, 419 (1992).

[8] P. Neu and R. Speicher, Z. Phys. B 95101 (1994). 
[9] S. Nakajima, Progr. Theor. Phys. 20, 948 (1958); R. Zwanzig, J. Chem. Phys. 33, 1338 (1960); H. Mori, Progr. Theor. Phys. 33, 127 (1965).

[10] R. H. Terwiel, Physica 74, 248 (1974).

[11] K. Mayurama and F. Shibata, Physica A 149, 447 (1988).

[12] H. K. Hartline, in Studies on Excitation and Inhibition in the Retina, edited by F. Ratcliff (Rockefeller University Press, New York, 1974).

[13] S. Kuffler and J. Nicholls, From Neuron to Brain (Sinauer, Sunderland, MA, 1977), pp. 31 and 32.
[14] J. G. Taylor, Neural Networks 3, 171 (1990).

[15] G. von Bekesy, J. Opt. Soc. Am 50, 1060 (1975).

[16] S. A. Shamma, J. Acoust. Soc. Am. 78, 1612 (1985).

[17] G. B. Ermentrout and J. D. Cowan, SIAM J. Appl. Math. 38, 1 (1980).

[18] I. Morishta and A. Yajima, Kybernetic 11, 154 (1972).

[19] H. Sumi, J. Chem. Phys. 67, 2943 (1977).

[20] S. Grossberg, Neural Networks 1, 17 (1988).

[21] L. F. Abbott, Network 2, 245 (1991). 